\title{
Antimicrobial Efficacy of Simarouba glauca (Lakshmi Taru) Plant Extract against Enterococcus faecalis Biofilm: An in vitro Study
}

\author{
${ }^{1}$ Sree L Varada, ${ }^{2}$ Jenaki E Veetil, ${ }^{3}$ Ganesh C Nair
}

\begin{abstract}
Aim: The aim of this study was to assess the antimicrobial activity of Simarouba glauca plant extract as intracanal medicament against Enterococcus faecalis and to compare its effect with that of metapex (calcium hydroxide with iodoform paste).

Materials and methods: In the study, 30 human premolar teeth infected with $E$. faecalis were used. Contaminated teeth were randomly separated into three groups $(n=10)$ and treated as follows: Group I, calcium hydroxide-based medication; group II, S. glauca-based medication; and group III, contaminated teeth without medication. After 7 days of treatment, teeth from each group were assessed for the level of microbial growth after a period of treatment.
\end{abstract}

Results: Metapex and S. glauca were associated with a reduction in E. faecalis after removal of the dressing; however, saline is associated with an increase in colony-forming units (CFU) per milliliter.

Conclusion: In this laboratory study using extracted teeth, S. glauca (Lakshmi Taru) was able to significantly reduce the CFU per milliliter values of $E$. faecalis in the root canal system similar to metapex.

Keywords: Enterococcus faecalis, Metapex, Phytotherapy, Simarouba glauca.

How to cite this article: Varada SL, Veetil JE, Nair GC. Antimicrobial Efficacy of Simarouba glauca (Lakshmi Taru) Plant Extract against Enterococcus faecalis Biofilm: An in vitro Study. Cons Dent Endod J 2017;2(2):43-47.

Source of support: Nil

Conflict of interest: None

\section{INTRODUCTION}

The goal of endodontic treatment is complete disinfection of complex root canal system, thereby preventing

\footnotetext{
${ }^{1,2}$ Postgraduate Student, ${ }^{3}$ Professor and Head

${ }^{1-3}$ Department of Conservative Dentistry and Endodontics, Sri Sankara Dental College, Thiruvananthapuram, Kerala, India

Corresponding Author: Sree L Varada, Postgraduate Student Department of Conservative Dentistry and Endodontics, Sri Sankara Dental College, Thiruvananthapuram, Kerala, India Phone: +914702795016, e-mail: sreelekshmi123sree@gmail. com
}

treatment failures and apical periodontitis. ${ }^{1}$ Bacterial colonization in root canal is difficult to eradicate by routine cleaning and shaping procedures.

Enterococcus faecalis is a facultative anaerobic Grampositive bacterium that has been isolated in high proportions in endodontic failures. They can invade the dentinal tubules to a depth of 300 to $1500 \mu \mathrm{m}$ and can resist antimicrobial agents used during treatment and endure potential starvation in cleaned and obturated canal. ${ }^{2}$

Intracanal medicaments help to eliminate or reduce microorganisms that remain after mechanical preparation so as to improve predictability and prognosis of treatment. ${ }^{3}$ Calcium hydroxide introduced by Herman in 1920 is routinely used as an intracanal medicament. The mechanism involves diffusion of hydroxyl ions in concentrations to reach adequate $\mathrm{pH}$ levels. ${ }^{4}$ Due to limited antibacterial efficiency of calcium hydroxide, several works have studied the mixture of other substances to calcium hydroxide. ${ }^{3}$

Metapex is silicon oil-based calcium hydroxide paste containing $38 \%$ iodoform. ${ }^{5}$ It has a $\mathrm{pH}$ below that which is effective to kill E. faecalis. The superior antimicrobial effects of metapex may be due to the combination with iodoform and to the viscous and oily vehicle, which may prolong the action of the medicament. ${ }^{6}$

The oily vehicles increase the antimicrobial effects of calcium hydroxide against $E$. faecalis and other bacteria. The use of iodoform and calcium hydroxide combination has been proved to be highly microbicidal due to synergistic effect. ${ }^{1}$

The superior antibacterial effects were demonstrated at dentinal tubule depths of up to $250 \mu \mathrm{m} .{ }^{5}$ The strong bactericidal properties of iodoform paste have been demonstrated in previous studies. ${ }^{7,8}$ Iodoform is effective as a disinfectant; it has been shown to be biocompatible with human periapical tissues and is often used as a resorbable dressing in pulpectomies of infected primary teeth. ${ }^{9,10}$ In the treatment or retreatment of persisting apical periodontitis, metapex may be the medicament of choice for the elimination of E. faecalis from the dentinal tubules. ${ }^{5}$

Simarouba glauca is commonly known as "Lakshmi Taru" or "paradise tree" belonging to the family of 
Simaroubaceae. It has a long history in herbal medicine in many countries. The bark and leaf extract of Simarouba is well known for its different types of pharmacological properties, such as hemostatic, antihelminthic, antiparasitic, antidysentric, antipyretic, and anticancerous. ${ }^{4}$

The aim of this study was to assess the antimicrobial activity of $S$. glauca plant extract as intracanal medicament against E. faecalis and to compare its effect with that of metapex (calcium hydroxide and iodoform paste).

\section{MATERIALS AND METHODS}

\section{Preparation of Plant Extract}

The leaves of S. glauca plant were dried in shade and powdered well. The powder was mixed with $200 \mathrm{~mL}$ of ethyl acetate and shaken well in a conical flask for 48 hours. Then, $100 \mathrm{mg}$ of extract is added to $400 \mathrm{mg}$ of polyethylene glycol in $5 \mathrm{~mL}$ of distilled water and stirred for 2 hours; $500 \mathrm{mg}$ of carbopol in $5 \mathrm{~mL}$ of distilled water was prepared and to which the extract was added simultaneously while stirring (Fig. 1A).

\section{Tooth Selection and Biomechanical Preparation}

Thirty freshly extracted single-rooted premolars were selected. The tooth crowns were sectioned using a highspeed motor and cylindrical diamond drill under cooling to standardize the length of each specimen at $14 \mathrm{~mm}$. The root canals were minimally instrumented using Gates Glidden drill sizes 1 and 2, K files 10 and 15, and with ProTaper F2 rotary system up to $1 \mathrm{~mm}$ short of the root apex. Root canal irrigation with $2 \mathrm{~mL}$ of $1 \% \mathrm{NaOCl}$ was carried out at each file change. Following this, canals were irrigated with $17 \%$ ethylenediaminetetraacetic acid (EDTA) for 3 minutes and then with $5 \mathrm{~mL}$ of saline solution. Afterward, apical foramen was sealed with light-cure composite resin, and the canals were dried with absorbent paper cones and autoclaved at $121^{\circ} \mathrm{C}$ for 20 minutes.

\section{Inoculation of $E$. faecalis}

The procedures were carried out in a laminar flow chamber (clean air systems CAH600). Test bacteria included E. faecalis American Type Culture Collection (29212). The culture bacterial suspension contained $1 \times 10^{7}$ $\mathrm{CFU} / \mathrm{mL}$ of $E$. faecalis.

Root canals were contaminated with this mixture. A sterile cotton pellet was placed at the root canal entrance. The microplates containing specimens were kept in microaerophilic environment at $37^{\circ} \mathrm{C}$ for 14 days (Fig. 1B). Brain heart infusion broth was applied to the root canals on alternate days. The root canal samples were harvested using sterile paper points to confirm contamination and transferred to tubes containing sterile saline. The contents of each canal were serial diluted and plated on anaerobic blood agar. The CFUs were counted after 24 hours of incubation (Fig. 2A).

\section{Division of the Experimental Groups}

The microplates containing the roots were randomly divided according to the medication used:

- Group I: Metapex group

- Group II: S. glauca group

- Group III: Without intracanal medication

After confirmation of bacterial contamination in the experimental groups, root canals were reprepared and irrigated with $5 \mathrm{~mL}$ of sterile saline. Following this, canals were irrigated with 17\% EDTA for 3 minutes and then with $5 \mathrm{~mL}$ of saline solution. After that root canals were dried with sterile paper points and filled with intracanal medicaments.

Sterile cotton pellets were placed at the root canal access cavities and specimens were incubated in microaerophilic environment for 7 days.

\section{Microbiological Analysis after Treatment}

After the dressings had been in place for 7 days, teeth from each group were irrigated with $5 \mathrm{~mL}$ of the
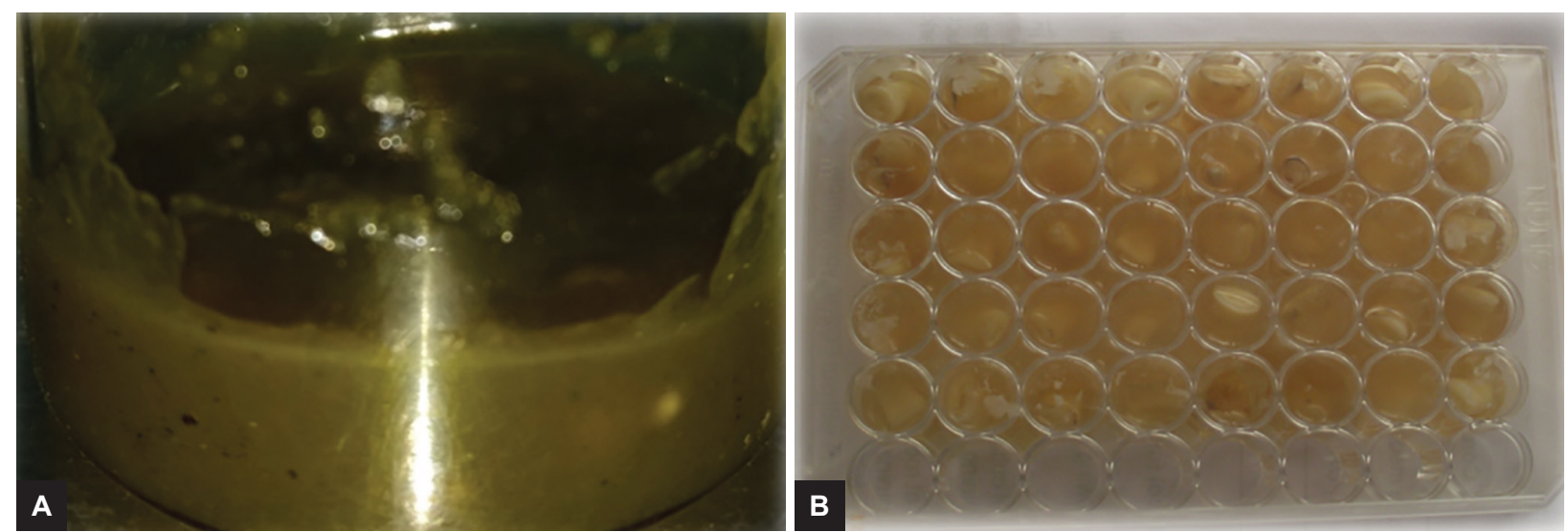

Figs 1A and B: (A) Prepared gel of Lakshmi Taru; and (B) inoculation with E. faecalis 

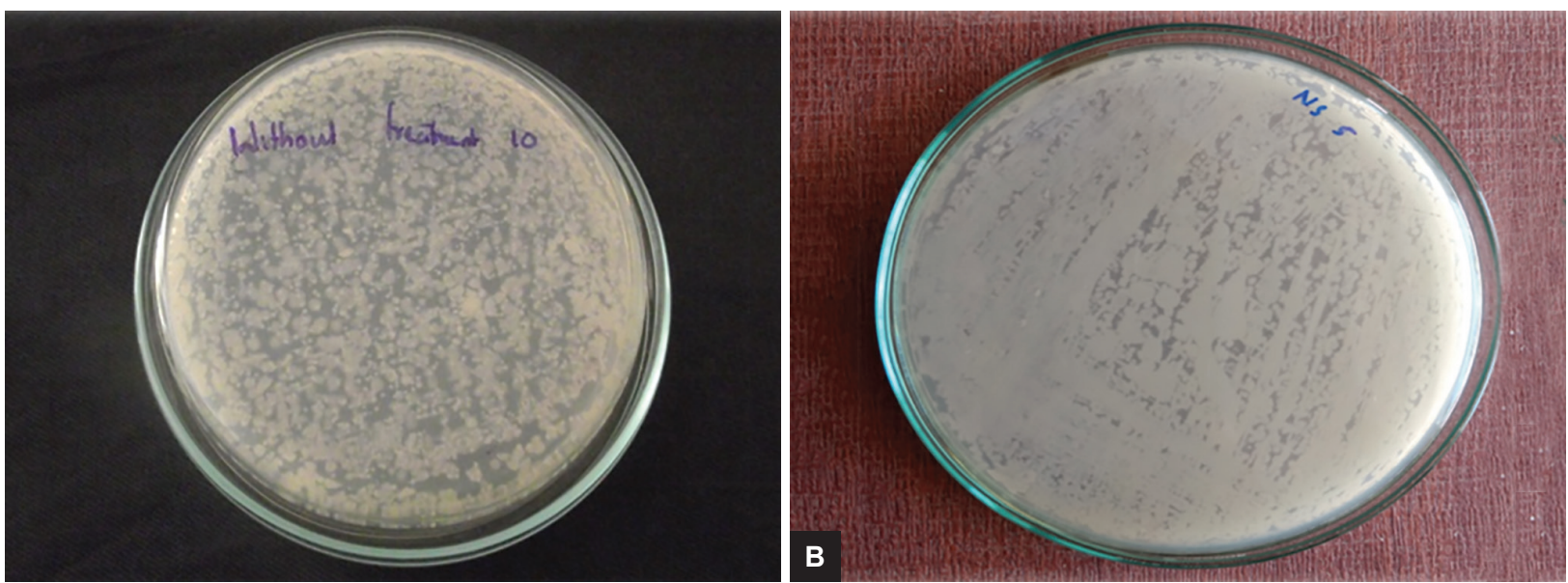

Figs 2A and B: (A) Premedication harvest; and (B) postmedication harvest without any medicament (saline)
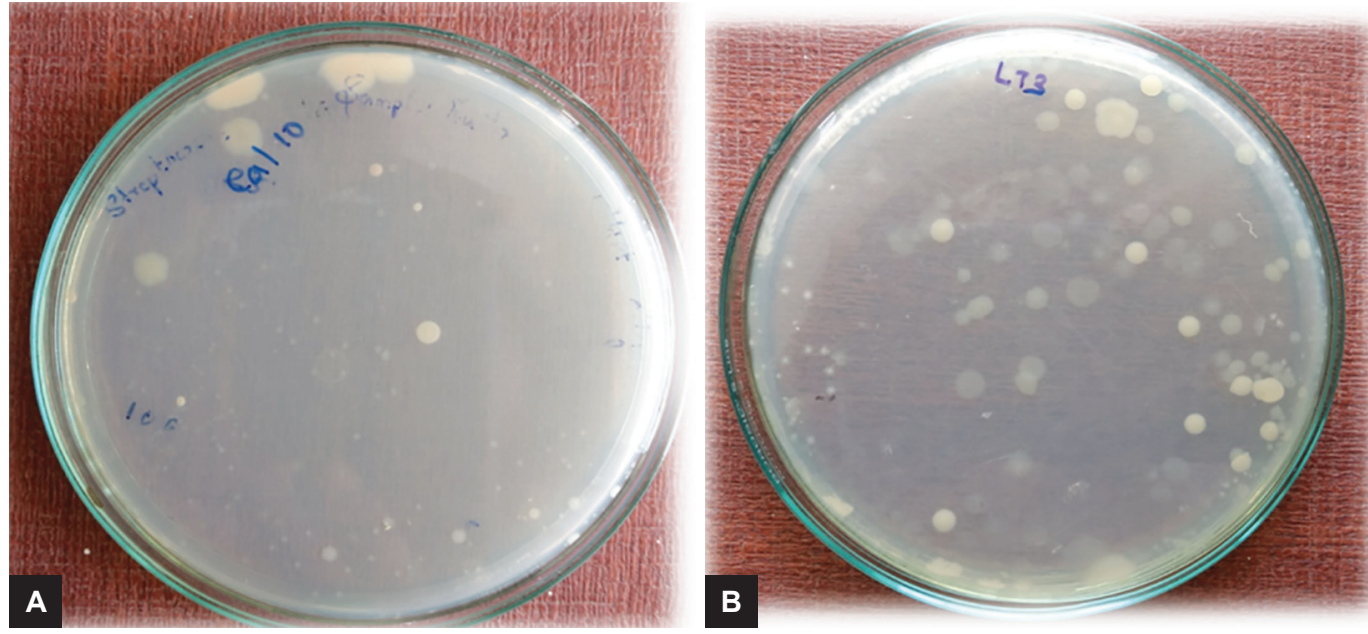

Figs 3A and B: (A) Postmedication harvest with metapex (A);and (B) S. glauca

sterilized saline solution, and a sterilized absorbent paper point was inserted into each root canal. The paper point was transferred to a test tube with sterile saline solution. The contents of each canal were serial diluted and plated on anaerobic blood agar. The CFUs were counted after 24 hours of incubation.

\section{Statistical Analysis}

Comparison between the experimental groups was carried out by Scheffe multiple comparison method. Comparison was between the control group and experimental groups using paired t-test. Significance level was set at $\mathrm{p}<0.05$.

\section{RESULTS}

The bacterial contamination was detected by recovering pure E. faecalis strains from all teeth at the initial harvest performed 14 days after incubation, which showed similar CFU/mL counts for all groups (Fig. 2B). After treating with intracanal medicaments, such as Lakshmi Taru, metapex postmedication harvest was taken, which showed reduction in the CFUs (Fig. 3). Specimens without intracanal medicaments (saline) confirmed the viability of $E$. faecalis throughout the study.

Groups I and II had lowest CFUs $/ \mathrm{mL}(\mathrm{p}<0.05)$ at the final harvest. Group III showed highest CFUs/ $\mathrm{mL}(\mathrm{p}<0.05)$ with no statistically significant difference between groups I and II, as shown in Table 1.

\section{DISCUSSION}

The prognosis of endodontic treatment is based on the disinfection of root canal system. ${ }^{2}$ The methodology used in this study simulated the clinical conditions in teeth with infected root canals, in which microorganisms may harbor in the areas of difficult access for the root canal preparation ${ }^{2}$ and medicaments with excellent coronal and apical seal. ${ }^{2}$ This study investigated the disinfection potential of $S$. glauca as an intracanal medicament.

Enterococcus faecalis was chosen because it is a facultative anaerobic microorganism present in root canal system and it has resistance to calcium hydroxide medicamentbased intracanal dressings. ${ }^{11}$ Enterococcus faecalis is associated with persistent apical periodontitis and resist 
Table 1: Comparison of pre- and posttest values in different groups

\begin{tabular}{|c|c|c|c|c|c|c|c|}
\hline Group (CFU/mL) & Stage & Mean & $S D$ & $n$ & Mean difference & Paired $t$ & $p$-value \\
\hline \multirow[t]{2}{*}{ Simarouba glauca } & Pretest & $98,960.0$ & $19,092.5$ & 10 & $92,640.0$ & $14.58^{\star *}$ & 0 \\
\hline & Posttest & 6320.0 & 2782.8 & 10 & & & \\
\hline \multirow[t]{2}{*}{ Calcium hydroxide } & Pretest & $92,620.0$ & $17,180.5$ & 10 & $88,884.0$ & $15.83^{\star *}$ & 0 \\
\hline & Posttest & 3736.0 & 2182.8 & 10 & & & \\
\hline \multirow[t]{2}{*}{ Saline (control) } & Pretest & $96,480.0$ & $18,250.6$ & 10 & $31,360.0$ & $3.92^{* *}$ & 0.004 \\
\hline & Posttest & $65,120.0$ & $13,762.2$ & 10 & & & \\
\hline
\end{tabular}

**Significant at 0.01 level; SD: Standard deviation

Table 2: Simarouba glauca composition

\begin{tabular}{ll}
\hline Contents & Active constituents \\
\hline Alkaloids & Ailanthone \\
Flavonoids & Benzoquinone \\
Cardenolides & Vacanthin \\
Glycosides & Dehydro-glaucarubinone \\
Phenolic compounds & Glaucarubin \\
Saponins & Glaucarubolone \\
Fixed oils & Holacanthone \\
& Melianone \\
& Simaroubidin \\
& Simarolide \\
& Simarubin \\
\hline
\end{tabular}

elimination from root canals. ${ }^{11}$ This microorganism has been used in several studies. ${ }^{4}$ The incubation period of 14 days allowed the suspension of microorganism to diffuse throughout the root canal system. ${ }^{5}$ The results revealed that medicaments tested reduced $E$. faecalis from the root canal system after removal of the dressing.

Metapex was used in this study because of its superior properties when compared with other calcium hydroxide-containing intracanal medicaments. Iodoform is incorporated to increase the antimicrobial properties of the material. Silicone oil acts as a vehicle. The mechanism of action can be described by the influence of $\mathrm{pH}$ on growth, metabolism, and bacterial cell division. The superior antimicrobial effects of metapex may be due to the combination with iodoform and to the viscous and oily vehicle which may prolong the action of the medicament. $^{6}$

Simarouba glauca (Lakshmi Taru) is a tree with various medicinal values for the past few years. All the parts of this plant is used in some way or another. It is widely used as a medicine for the treatment of various diseases, like cancer, amoebiasis, ulcer, and diarrhea. ${ }^{12}$
Table 3: Effectiveness of medicaments in reducing bacterial $\mathrm{CFU} / \mathrm{mL}$

\begin{tabular}{llllll}
\hline Group (CFU/mL) & Mean & $S D$ & $n$ & $f$-value & $p$-value \\
\hline Simarouba glauca & $98,960.0$ & $19,092.5$ & 10 & & \\
Metapex & $92,620.0$ & $17,180.5$ & 10 & 0.31 & 0.737 \\
Saline & $96,480.0$ & $18,250.6$ & 10 & &
\end{tabular}

SD: Standard deviation

The alkaloids present in plant extract have alkaline $\mathrm{pH}$ (Table 2). They could affect the growth and metabolism of E. faecalis. So their pharmacological actions may be the reason for the antibacterial property of S. glauca. ${ }^{12}$

A study was done using S. glauca plant extract as intracanal irrigants. It was concluded that the irrigants could reduce the number of CFUs of E. faecalis per milliliter. But the effectiveness was not as good as chlorhexidine. In this study, plant extract was used as medicaments and it was found that the medicament could reduce the amount of microorganism more than when it was used as intracanal irrigant.

In this study, a gel was prepared from the extract of S. glauca plant; here vehicle is carbopol. It was used as a medicament in biomechanically prepared extracted teeth, and the effectiveness in the removal of E. faecalis was determined along with metapex. An incubation period of 14 days within the root canal system effectively increased its action. Colony forming units per milliliter of $E$. faecalis were calculated before and after placing medicaments.

The CFU of E. faecalis per milliliter was calculated without using medicaments (Table 3). There was no significant difference in the amount of colonies in experimental groups and control group. The CFUs of E. faecalis per milliliter was calculated after the use of S. glauca, metapex in groups I and II respectively. The CFUs / $\mathrm{mL}$ of E. faecalis in S. glauca group was compared with metapex group and saline group. Similarly, metapex group was compared with saline group (Table 4 ). There was no

Table 4: Comparison of posttest value based on group

\begin{tabular}{lllllllll}
\hline & & & & & & Scheffe multiple \\
Group (CFU/mL) & Mean & SD & $n$ & $f$-value & Sig. & comparison pair & $f$-value & $p$-value \\
\hline Simarouba glauca (A) & 6320.0 & 2782.8 & 10 & & & A and B & 0.2 & 0.782 \\
Metapex (B) & 3736.0 & 2182.8 & 10 & $179.1^{* *}$ & 0 & A and C & $128.4^{* *}$ & 0 \\
Saline (control) (C) & $65,120.0$ & $13,762.2$ & 10 & & & B and C & $140^{* *}$ & 0 \\
\hline
\end{tabular}

**Significant at 0.01 level; SD: Standard deviation 


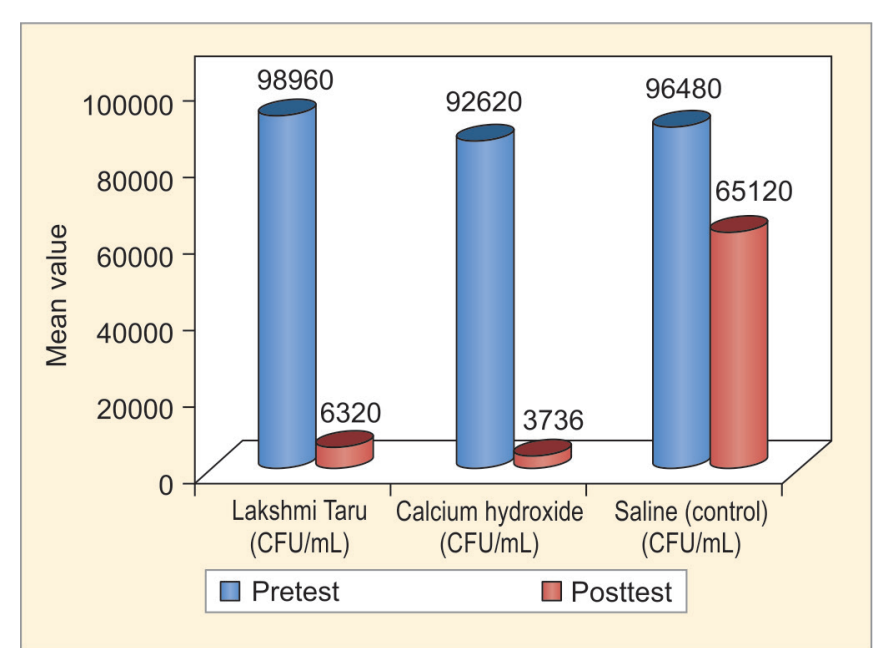

Graph 1: Comparison of effectiveness of medicaments in reducing bacterial CFU unit/mL

significant difference in the reduction of CFUs. Here it was found that $E$. faecalis colonies were effectively reduced with the use of metapex.

When comparing pretest and posttest values in S. glauca, paired t-test was used for the comparison and it was found that the values are significant at 0.01 level. Comparing pretest and posttest values of $\mathrm{CFU} / \mathrm{mL}$ of saline, there was no significant reduction in the amount of E. faecalis. This clearly indicated that $S$. glauca was effective in reducing the $\mathrm{CFU}$ of microorganisms per milliliter and was as effective as metapex.

The pretest and posttest values of CFUs/mL were compared (Graph 1), and it was found that there was a good reduction in the amount of E. faecalis in both metapex and S. glauca group without any statistically significant difference. In saline group, there was no reduction in the amount of CFUs in the pretest and posttest groups.

The alkaline nature of S. glauca extract could be the reason for its antimicrobial activities. The future of herbal intracanal medicaments hold a promising effect in the reduction of bacterial colonies within the root canal system.

\section{CONCLUSION}

Based on the findings obtained, it is possible to conclude that $S$. glauca leaf extract is efficient in reducing endodontic microorganisms as comparable to metapex. The alkaloids present in Lakshmi Taru could be able to enhance the antimicrobial property, by inhibiting the growth and metabolism of E. faecalis. Further investigations are required to know about the efficacy of $S$. glauca against multispecies biofilm and mechanisms inside the root canal system as well as the need to associate it with other medicaments. So phytotherapy is a viable option for endodontic management.

\section{REFERENCES}

1. Lima RK, Guerreiro-Tanomaru JM, Faria-Júnior NB, Tanomaru-Filho M. Effectiveness of calcium hydroxide-based intracanal medicaments against Enterococcus faecalis. Int Endod J 2012 Apr;45(4):311-316.

2. Cwikla SJ, Bélanger M, Giguère S, Progulske-Fox A, Vertucci J. Dentinal tubule disinfection using three calcium hydroxide formulations. J Endod 2005 Jan;31(1):50-52.

3. Estrela C, Rodrigues de Araújo Estrela C, Bammann LL, Pecora JD. Two methods to evaluate the antimicrobial action of calcium hydroxide paste. J Endod 2001 Dec;27(12): 720-723.

4. Patil Manasi S, Gaikwad DK. A critical review on medicinally important oil yielding plant laxmitaru (Simarouba glauca DC.). J Pharm Sci Res 2011 Apr;3(4):1195-1213.

5. Gomes BP, Ferraz CC, Garrido FD, Rosalen PL, Zaia AA, Teixeira FB, de Souza-Filho FJ. Microbial susceptibility to calcium hydroxide pastes and their vehicles. J Endod 2002 Nov;28(11):758-761.

6. Evans M, Davies JK, Sundqvist G, Figdor D. Mechanisms involved in the resistance of Enterococcus faecalis to calcium hydroxide. Int Endod J 2002 Mar;35(3):221-228.

7. Haenni S, Schmidlin PR, Mueller B, Sener B, Zehnder M. Chemical and antimicrobial properties of calcium hydroxide mixed with irrigating solutions. Int Endod J 2003 Feb;36(2):100-105.

8. Portenier I, Happasalo H, Rye A, Waltimo T, Ørstovik D, Hapasalo M. Inactivation of root canal medicaments by dentin hydroxyapatite and bovine serum albumin. Int Endod J 2001 Apr;34(3):184-188.

9. Manzur A, González AM, Pozos A, Silva-Herzog D, FriedmanS. Bacterial quantification in teeth with apical periodontitis related to instrumentation and different intracanal medications: a randomized clinical trial. J Endod 2007 Feb;33(2): 114-118.

10. Portenier I, Waltimo TM, Haapasalo M. Enterococcus faecalis - the root canal survivor and 'star' in post-treatment disease. Endod Topics 2003 Nov;6(1):135-159.

11. Gautam S, Rajkumar B, Landge SP, Dubey S, Nehete P, Boruah LC. Antimicrobial efficacy of metapex (calcium hydroxide with iodoform formulation) at different concentrations against selected microorganisms - an in vitro study. Nepal Med Coll J 2011 Dec;13(4):297-300.

12. Atila-Pektas B, Yurdakul P, Gülmez D, Görduysus O. Antimicrobial effects of root canal medicaments against Enterococcus faecalis and Streptococcus mutans. Int Endod J 2013 May;46(5):413-418. 